\title{
Paradoxes de l'identité : de la déconstruction à la construction identitaire dans Les petits garçons naissent aussi des étoiles d'Emmanuel Boundzeki Dongala
}

\author{
Moussa Coulibaly \\ Université de Cocody-Abidjan
}

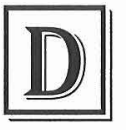

e la différence sexuelle, ans le domaine littéraire en général, et dans le roman africain en particulier, la question de l'identité n'est pas ignorée. Elle a fait et continue de faire l'objet de nombre de travaux de réflexion. Cependant, sa dimension qui semble plus en vogue dans le roman africain est celle donnée par l'anthropologie où l'identité est perçue sous deux aspects : personnel et culturel. Ces deux aspects vont nous préoccuper dans le cadre de notre réflexion menée à partir de Les

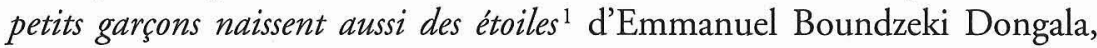
roman dans lequel l'identité se perçoit comme l'image résultant des interactions entre les individus, les groupes, la société et leurs idéologies. Elle se construit et constitue pour l'individu ou les individus un cadre psychologique (schéma mental et système de représentation).

Dans le roman de Dongala, l'individu renvoie au personnage central, narrateur dans l'œuvre; le groupe à la famille et enfin, la société à la grande communauté comprenant les univers religieux, scientifique, traditionnel. Ce sont ces ensembles qui, dans leurs interactions, déconstruisent une identité inacceptable dans leur entendement. Or ce faisant, ils créent sans toutefois le savoir une nouvelle identité. D'où justement l'idée de paradoxes liée à la création (construction) d'identité dans ce roman. Si nous convenons que paradoxe s'entend ici par " ce qui est en contradiction avec la logique, avec le bon sens ", alors on est en droit d'affirmer que le roman Les petits garçons naissent aussi des étoiles traduit parfaitement cette contradiction, du moins ce paradoxe identitaire qui, en niant une identité en crée une autre.

1 Emmanuel Boundzeki Dongala, Les petits garçons naissent aussi des étoiles, Paris, Le serpent à plumes, 1998. 
Notre analyse bâtie autour de ce paradoxe présentera d'abord la crise de l'identité gémellaire comme fondement de ce paradoxe identitaire, ensuite les interactions sociales dont l'impact conduit à la re-construction identitaire involontaire et enfin la survalorisation de l'identité personnelle construite.

\section{La crise de l'identité gémellaire.}

Dans Les petits garçons naissent aussi des étoiles, la diégèse commence par un fait insolite, une naissance de jumeau jamais vue, qui suscite beaucoup de réactions allant jusqu'à une remise en cause de cette identité gémellaire. Il se crée ainsi un mythe de cette extraordinaire gémellité; lequel mythe est perçu comme le fait de certaines institutions qui prennent pour fondement de leurs agissements une certaine logique ou philosophie communautaire.

\section{LE MYTHE D'UNE EXTRAORDINAIRE GÉMELLITÉ}

La gémellité dans le roman de Dongala concerne trois personnages dont le narrateur lui-même ${ }^{2}$. Si les deux premiers sont nés le même jour et à intervalle de temps raisonnable, donc conforme à la venue au monde de jumeaux, il n'en a pas été de même pour le troisième Matapari, né quarante-huit heures après eux. C'est sur ce dernier né que se fonde tout le paradoxe identitaire. Issus de la même grossesse, ces trois garçons sont en principe des triplés, une identité que nul ne saurait leur contester. Mais l'extraordinaire parturition de Matapari fait de lui un être exceptionnel et sa naissance ne trouve pas d'explication sociologique, ni culturelle (c'està-dire traditionnelle), ni religieuse encore moins scientifique. On assiste alors progressivement à la naissance ou à la création d'un mythe de cette gémellité sans pareille. Le mot mythe recouvre plusieurs acceptions dont celle de Mircea Eliade que voici :

"Le mythe raconte une histoire sacrée; il relate un événement qui a eu lieu dans le temps primordial, le temps fabuleux des commencements [...] C'est le récit d'une création : on raconte comment quelque chose a été produit, a commencé à être ${ }^{3}$... »

À considérer cette définition de Mircéa Eliade, les aspects tels : histoire sacrée, un événement qui a eu lieu, récit d'une création, comment quelque

2 Il s'agit de Banzouzi et Batsimba, les deux premiers nés et Matapari le troisième né.

3 Mircéa Eliade, Aspects du mythe, Paris, Gallimard, 1963, p. 16-17. 
chose a été produit, et a commencé à être peuvent se rapporter à la naissance du narrateur personnage Matapari, le troisième né des jumeaux; une naissance qui passe pour être une exception. Elle a été présentée comme un événement qui ne s'est jamais produit, une chose curieuse que la tradition ignore, un fait sacré.

L'événement produit revêt une dimension sacrée. Il se présente comme une histoire sacrée, un événement qui a eu lieu. C'est en somme le récit d'une création, fruit de l'imaginaire populaire et qui raconte comment quelque chose, un fait peu commun a été produit, a commencé à être... C'est donc le mythe d'une gémellité.

Le personnage Matapari n'est plus vu comme un jumeau avec ses deux frères jumeaux qui font avec lui des triplés, mais il est devenu un Etre présenté comme "Surnaturel » grâce à qui " une réalité est venue à l'existence, $[. .$.$] un comportement bumain, une institution { }^{4}$ "

L'imaginaire populaire, fortement ancré dans la culture traditionnelle africaine trouve une explication mythique, énigmatique à la naissance du jumeau. Pour lui en fait, il y a eu deux naissances distinctes : celle des vrais jumeaux, les vrais que la tradition admet et que la science même explique ${ }^{5}$, et celle de Matapari le narrateur qui ne trouve d'explication nulle part ailleurs que dans la superstition. Qui est donc ce personnage, ce jumeau qui semble l'être sans toutefois l'être? Si son identité gémellaire est remise en cause, certaines institutions y contribuent fortement.

\section{LE RÔLE DES INSTITUTIONS}

Le terme institution renvoie ici à deux réalités à savoir la famille et la religion. Celles-ci ont eu une réelle emprise sur le mental populaire à telle enseigne que le processus de déconstruction de l'identité gémellaire a été rendu non seulement possible mais aussi facile.

Dans Les petits garçons naissent aussi des étoiles, le phénomène gémellaire engendré par la naissance énigmatique de Matapari a entraîné une bipolarisation de la cellule familiale. On note d'un côté le père qui

4 Ibidem, p. 16-17.

5 Le terme jumeau se dit des enfants (deux ou plusieurs) nés d'un même accouchement. Biologiquement, la science distingue deux sortes : les vrais jumeaux, nommés jumeaux univitellins ou monozygotes, sont issus de la division précoce d'un œuf; toujours du même sexe, ils se ressemblent physiquement et psychiquement, ont la même résistance aux maladies, etc. Les faux jumeaux, ou bivitellins, ou dizygotes, issus de deux oufs différents, peuvent être de sexe différent et fort dissemblables. 
cherche une explication scientifique à cette naissance et de l'autre, la mère et son clan enfermés dans leurs superstitions. Face à ces deux perceptions des choses, voire à ces deux conceptions diamétralement opposées d'une même réalité, l'identité gémellaire du narrateur Matapari est récusée. À vrai dire, le clan maternel, par ses agissements et ses discours, fait de l'identité gémellaire de Matapari une identité qui étouffe et déconstruit son identité vraie, celle de triplé pour en faire " un mal aimét ", un indésirable dont la venue au monde ébranle la quiétude familiale et sociale. Il n'est donc pas le bien venu parmi eux. À cette position familiale s'allie l'opinion religieuse.

On note effectivement, dans ce processus de déconstruction identitaire du nouveau-né, l'entrée en scène de deux religieux et non des moindres : Konaté, grand musulman et Boniface, prêtre catholique. Tous les deux voient en cet événement, la manifestation du Satan qu'il faut absolument chasser. Cependant, si l'échec de leur entreprise est notoire, l'effet produit dans le clan des superstitieux est spectaculaire. Il les conforte dans leur position. Matapari n'est plus considéré comme l'enfant de sa propre mère mais comme un tout autre enfant. "Rejeté " ainsi par nombre de personnes dont sa mère et ses frères jumeaux, sa personnalité humaine et surtout gémellaire se trouve aussi niée. Si la crise identitaire dont il est question a été partiellement favorisée par une partie de la famille et soutenue par les religions, une certaine logique communautaire a servi à l'entretenir.

\section{La logique communautaire}

La logique communautaire sous-entend la tentative de justification symbolique de la personnalité, mieux de l'identité gémellaire qu'est le triplé. Selon la logique culturelle de cette communauté, les jumeaux portent un nom qui est fonction de leur ordre de naissance. On les nomme toujours «Banzouzi » pour le premier sorti et «Batsimba» pour le second au dire du narrateur. Il en va de même pour celui qui les suit " $d e$ Milandou ». Or le narrateur personnage ne s'inscrit dans aucun de ces cas du fait de l'écart temporel de quarante-huit heures qui sépare sa naissance de celle des deux autres jumeaux. Cet écart constitue un mystère auquel nul ne peut trouver d'explication.

Matapari est alors indéfinissable aux yeux de la communauté; d'où son sobriquet "Matapari, enfant à problème ». Le personnage mystérieux, insaisissable qu'il est, suscite ainsi au sein de la communauté des réactions

6 Néologisme créé par Guillaume Apollinaire sur le modèle de «bien aimé » et qui sert de titre à un de ses poèmes dans Alcool. Cette expression traduit ici la situation que vit Matapari. 
négatives contribuant également au processus de déconstruction identitaire. Ce processus est soutenu par la superstition, croyance populaire qui endoctrine l'esprit communautaire et selon laquelle tout fait, tout événement survenu doit pouvoir trouver une explication culturelle, sociologique, traditionnelle. Face à cette logique de pensée, l'identité première de Matapari est récusée pour faire place à plusieurs attributs dont on l'affuble. La scène d'exorcisme de Mama Kossa révèle cet esprit de superstition communautaire :

" Mais petit, tu as affaire à Mama Kossa [...], je te protégerai contre les gens et je protégerai les gens contre toi. [...] enfant surprise, $[\ldots]$ enfant esprit des eaux et des forêts, [...] enfant-ancêtre qui nous est revenu... ". (p. 26-27)

Cette scène d'exorcisme atteste du rejet pur et simple du personnage. Toutes ces agitations de la communauté traduisent les toutes premières articulations possibles de sa perception et de sa compréhension des êtres et du monde. C'est grâce à cette conception des choses que la communauté donne sens à tout ce qui constitue l'univers sémantique de la personnalité gémellaire. Dès lors, dans l'appréhension physique du monde environnant, ou dans les constructions mentales qu'élabore cette communauté, l'identité gémellaire, en l'occurrence celle présentée dans le roman de Dongala qui retrace la vie d'un personnage, amorce une phase de modification ou de transformation. Et comme l'atteste si bien Joseph Courtés, "pour le lecteur, et ceci est une condition sine qua none de la compréhension d'un tel livre, ledit personnage demeure toujours lui-même et, en même temps, subit des transformations $^{7}$. " Au regard de tout ce qu'a engendré la crise gémellaire dans ce roman, il ressort que, en tentant de saisir une personnalité gémellaire très contestée, les divers comportements et agissements ont créé un phénomène inattendu, involontaire, celui du paradoxe de l'identité. Lacte de déconstruction appelle celui de reconstruction de l'identité; ce que nous nous proposons d'analyser à présent.

\section{L'impact des interactions sociales : une reconstruction identitaire involontaire}

Dans Les petits garçons naissent aussi des étoiles de Dongala, les interactions sociales ont donné lieu à un paradoxe lié à l'appréhension identitaire. En

7 Joseph Courtés, Analyse Sémiotique du Discours, de l'énoncé à l'énonciation, Paris, Hachette, 1991, p. 69. 
détruisant une identité, l'acte contribue à la reconstruction d'identité à partir de la confrontation des structures tels le singulier et le collectif, du discours social et de la mise en scène du « soi » concerné.

\section{LE SINGULIER FACE AU COLLECTIF DANS LA CONSTRUCTION IDENTITAIRE}

Dans son récit, Dongala fait saisir la question de l'identité de son narrateur personnage à travers les interactions individuelles. Trois personnages : le père, la mère et le prêtre Boniface, par leurs actes préparent et conditionnent la problématique du désir d'identification qui, plus est, se trouve associée à une sorte de loi symbolique. Le narrateur personnage revêt alors une identité symbolique. Et toute la question de son identité " réside désormais dans l'articulation entre le singulier et le collectif ${ }^{8}$ ".

Le singulier se réfere au père et le collectif à la mère et à ses alliés. Dès lors, le narrateur personnage n'est plus que ce que symboliquement le singulier et le collectif voudront qu'il soit. Or la volonté de ces deux entités (le singulier et le collectif) de l'identifier, dévoile une logique conflictuelle. Pour le collectif il est le "faux jumeau ", l'être de mauvais augure. Il est " admis » dans la société sans pourtant l'être en réalité. Son identité devenue problématique semble l'éloigner du reste social eu égard aux différents faits et gestes observés.

À l'opposé et paradoxalement se trouve une structure patriarcale très forte qui en niant et en réfutant les arguments du collectif, essaie de trouver une identité au narrateur. Ainsi, au faux jumeau se substitue le jumeau tout court, l'être de mauvais augure devient le sauveur. Et au lieu de Matapari, le père le prénomme Michel, prénom très symbolique ${ }^{9}$ qui va même fonctionner par la suite comme un impératif catégorique du narrateur personnage. Du coup, son programme narratif se trouve ainsi établi par le singulier avec pour rôle celui d'actant sujet sauveur comme le démontre la scène produite à l'hôpital. En posant sa main sur le front de son frère Banzouzi malade, celui-ci faillit s'étouffer puis émit un puissant éternuement :

" La graine qui était coincée dans sa narine fut projetée violemment comme un bouchon de champagne que l'on fait sauter [...]. » (p. 96-97)

8 Jacques Saliba, "De l'identité aux identités " in Champ psychosomatique : les identités, Paris, L'Esprit du Temps, 2001, p. 7.

9 Michel ou Michaël (saint), est un des archanges, chef de la milice céleste qui protège Israël, d'après le prophète Daniel (Daniel, X, 13). 
À partir de la guérison miraculeuse du frère jumeau, le processus de reconstruction identitaire du narrateur personnage est mis en branle. Son geste sauveur est la confirmation du rôle d'archange que le prénom Michel l'autorise à assumer. L'identité de Michel est alors construite progressivement dans le roman à travers le jeu de déconstructionconstruction. Et son identité recouvre ce champ des rapports humains où il s'efforce d'opérer une synthèse entre les forces internes et son action, entre ce qu'il est pour lui et ce qu'il est pour les autres. Comme son identité n'est pas obligatoirement accordée, mais s'inscrit plutôt dans un jeu de forces sociales dont le pôle dominant est la force paternelle, il y a lieu aussi de se référer au discours social.

\section{LE DISCOURS SOCIAL ET LA QUESTION DE LA RECONSTRUCTION IDENTITAIRE}

La tentative de déconstruction de la personnalité première du narrateur personnage a donné lieu paradoxalement à une reconstruction identitaire. Celle-ci est vue comme une résultante des enjeux de dénégation et d'affect. Elle n'est donc pas un acte nécessairement volontaire mais elle se présente plutôt comme la conséquence des différents discours tenus à l'endroit du personnage. Depuis la naissance de celui-ci on a constaté que les discours familial et social ont été des discours de dénégation qui ont accompagné son développement et lui ont attribué des identités diverses en vue de sa disqualification. Mais ces qualificatifs dépréciatifs qui, normalement avaient pour rôle de détruire la personnalité du narrateur ont plutôt produit un effet contraire. Une identité se déconstruit verbalement (discursivement) et donne lieu dans le même temps à une reconstruction d'une identité nouvelle. Ainsi "les identités sont des réalités d'ordre discursif, [...] susceptibles d'être déconstruites [...] mais aussi reconstruites ${ }^{10}$. "Dans ce roman, le discours anticipe et oriente la formation de l'identité; il assigne une place au personnage dans la constellation familiale en même temps qu'il le projette dans l'avenir à travers l'image suggérée de son destin futur comme prétend le savoir la société.

Ainsi en ce personnage se perçoit le choc des deux positions discursives, "se rencontrent des appartenances multiples qui sopposent parfois

10 Pierre Halen, "Constructions identitaires et stratégies d'émergence : notes pour une analyse institutionnelle du système littéraire francophone » in Études françaises, La littérature africaine et ses discours critiques, Montréal, Les Presses de l'Université de Montréal, vol. 37, n² 2, 2001, p. 26. 
entre elles et le contraignent ${ }^{11}$ " ̀̀ la recherche de sa vraie personnalité. Celleci, sans paraître un acte volontaire, se construit progressivement à l'ombre des différents discours qui se tiennent. En refusant l'image (l'identité) qu'un certain type de discours projette de lui et s'inscrivant en partie dans le discours du père et du grand-père, le narrateur personnage subit une métamorphose, une transformation progressive de sa personnalité. Il se présente comme un personnage de deux cultures : occidentale par le biais de la science et africaine par la formation traditionnelle initiée par le grandpère. À partir de cette double culture du narrateur personnage, Dongala nous le donne à lire comme un personnage investi d'une mission à accomplir. Celle-ci passe par une certaine affirmation du narrateur personnage au sein de sa communauté et révèle en même temps son identité personnelle.

\section{Construction sociale de l'identité personnelle DU NARRATEUR PERSONNAGE}

Le rejet du narrateur ou l'ostracisme dont il a été frappé dans le roman s'est avéré être, en fin de compte, un facteur déterminant dans la construction de sa personnalité. L'image qu'il donne de lui est celle qui fait de lui l'un des acteurs principaux de cette fiction romanesque. S'il a « failli ne pas être né » comme il se plaît à le dire dès les pages incipitales, on le découvre par la suite comme un sauveur, un grand combattant volant au secours des autres. En outre la coïncidence de sa naissance avec l'anniversaire de l'indépendance du pays n'est pas fortuite, elle est porteuse de sens. En effet, la déconstruction de l'identité du narrateur personnage est sémantiquement synonyme de la défiguration de la démocratie au sens vrai du terme qui, elle aussi a "failli ne pas être née ». En voulant étouffer la démocratie, on l'a plutôt aidée à naître. Ce paradoxe de la démocratie résume celui de l'identité du narrateur. Les différentes contradictions sociales, en ouvrant dans le sens de l'exclusion du narrateur, l'ont plutôt élevé intellectuellement, physiologiquement, psychologiquement. A ce titre, il devient, au-delà de sa simple personnalité, l'Afrique qui tient à ses valeurs, qui refuse de mourir; l'espoir d'une renaissance africaine. Il est perçu comme la cendre d'où jaillira cette nouvelle Afrique. C'est pourquoi son grand-père a trouvé nécessaire de lui enseigner la tradition. Le narrateur personnage se doit d'être la source inépuisable du savoir

11 Amin Maalouf, Les identités meurtrières, Paris, Éditions Grasset et Fasquelle, 1998, p. 10. 
(traditionnel et occidental), sa vie le germe des vies; les préjugés et convictions sur sa personne, le feu des idées et des actions nouvelles. Ainsi, la société dans son entièreté, aussi bien celle qui l'exclut que celle qui se donne pour tâche de l'édifier, a fait de lui ce qu'il est. L'être nouveau qu'il est devenu en fin de compte est le fruit des contingences sociales. Et tout le texte fonctionne en somme comme une survalorisation de son identité.

\section{La survalorisation de l'identité du narrateur personnage}

L'analyse de la question de déconstruction reconstruction identitaire dans Les petits garçons naissent aussi des étoiles n'est pas sans susciter celle de la valeur de l'identité. Étant donné que ce qui permet d'identifier un individu est en même temps ce qui le distingue de l'autre ou des autres, la survalorisation de l'identité suppose, a priori, la question de l'altérité et du soi. Le narrateur personnage, dans son évolution textuelle, donne une image valorisante de lui-même qui se perçoit par rapport aux autres jumeaux. Cette identité le définit et le met en scène. Dans cette optique, son identité recouvre une fonction ontologique et pragmatique. Le cas de Matapari donne aussi à Dongala l'occasion de prôner son idéal identitaire.

\section{LA QUESTION DE L'ALTÉRITÉ ET DU SOI ${ }^{\mathrm{I2}}$}

La question de l'altérité et du soi révèle et définit en fait l'identité personnelle réelle du narrateur personnage, donnée ici comme une notion complexe. Celle-ci revêt deux significations, objective et subjective.

Du point de vue objectif, le narrateur se présente comme un individu unique, différent de ses deux frères jumeaux par son patrimoine génétique.

12 Selon le sociologue américain George H. Mead, l'un des fondateurs de la psychologie sociale, et cité par Edmond Marc Lipiansky, il faut distinguer trois instances forgeant l'identité : le moi, le je et le soi. Le moi est l'ensemble des rôles que l'individu apprend à tenir dans la société qui est la sienne. Le comportement individuel ne peut donc être compris qu'en fonction du comportement collectif. Mais l'individu est également capable de spontanéité et d'innovation personnelle, ce qui est la fonction spécifique du je. Le soi (l'identité) est l'association entre ces deux éléments : le moi (intégration des normes sociales) et le je (actions spontanées). On pourrait alors comprendre à la suite de George H. Mead que le soi est la conceptualisation par la psychologie de la question "Qui suis-je? » Il peut être ainsi défini comme l'ensemble des éléments qui nous caractérisent c'est-à-dire la façon dont nous nous définissons. 
Mais cette identité a surtout un sens subjectif. Le sens subjectif lui, fait ressortir le sentiment d'individualité, de singularité du narrateur c'est-àdire qu'il est d'abord lui-même et ensuite différent des autres et a telles ou telles caractéristiques. Le texte dans son fonctionnement nous fait sortir de la similitude du narrateur personnage avec ses deux autres frères c'est-à-dire leur gémellité pour aller vers leur différence.

Dans cet élan de la similitude à la différence, on note dans la psychologie du narrateur personnage lui-même que son identité se construit dans un double mouvement d'assimilation et de différence, d'identification aux autres et de distinction par rapport à eux. Quand il dit à propos de ses frères Banzouzi et Batsimba qu'ils sont jumeaux entre eux et triplés avec lui, il met en relief cette similitude et cette différence. La façon de concevoir ses frères et lui-même amène le narrateur personnage à tenter d'une part, dans un effort inconscient, à rétablir sa solidarité avec ses frères et d'autre part, de façon consciente à montrer sa spécificité individuelle qui vient du regard que les autres portent sur sa personne. Par ses relations humaines, le narrateur personnage tente de faire reconnaitre aux autres la valeur sociale positive qu'il revendique. On comprend alors aisément que la question de l'identité personnelle ou du soi ne peut être séparée du concept d'altérité. Elle est vue comme un rapport et pas une qualification. Ainsi la question de "lidentité est non pas "qui suis-je?", mais "qui je suis par rapport aux autres, que sont les autres par rapport à moi $i^{13}$ ?" "L'altérité et le soi sont donc deux facettes intimement liées et nécessaires dans la compréhension et la définition de l'identité qui, somme toute, remplit une fonction.

\section{Fonction ONTOlogique ET PRAGMATIQUe de L'IDENTIté DU NARRATEUR}

Ces fonctions liées à l'identité du narrateur se rapportent à sa culture. Celle-ci n'est pas une empreinte inamovible, une donnée immuable sur laquelle s'appuyer pour fonder sa différence. "Les cultures sont des formations historiques évolutives (ce qui relativise la notion de "culture d'origine ") et qui de surcroît, doivent beaucoup à l'emprunt" ".

13 Il s'agit de propos tenus par J.-F. Gossiaux lors d'une conférence organisée par l'entreprise de diffusion des revues scientifiques DIF POP sur "L'identité nationale » en juin 1997 et repris par Jean-Claude Ruano-Borbalan dans l'introduction de la revue Sciences Humaines de 1998.

14 Carmel Camilleri, "Cultures et stratégies, ou les mille manières de s'adapter " in Revue Sciences Humaines : Lidentité, L’individu, Le groupe, La société, Auxerre, Éditions Sciences Humaines, 1998, p. 58. 
Dans le cas d'espèce, le narrateur de Les petits garçons naissent aussi des étoiles a été influencé par deux cultures presque antagonistes mais il a su éviter le conflit par une cohérence simple c'est-à-dire qu'il a pu préserver en lui le système culturel autour duquel il s'est édifié en évitant d'être totalement phagocyté par le système étranger qui a infiltré fortement son environnement. Ce faisant, il évite un conflit intérieur au détriment de son adaptation. En d'autres termes, il survalorise la fonction ontologique de son identité aux dépens de sa fonction pragmatique. En Matapari le narrateur personnage s'articule la représentation des deux cultures en présence. Son identité reflète l'élaboration des formations équilibrées (souci ontologique) et, dans le même temps, il s'adapte à l'environnement tel qu'il est devenu (souci pragmatique).

Le narrateur personnage de ce texte devient surtout l'image de ce que doit être l'Africain d'aujourd'hui, un Africain poly-identitaire dans l'entendement de Dongala, c'est-à-dire ancré dans sa culture et ouvert au reste du monde. Car comme le souligne si bien Carmel Camilleri, il faut "signifier la différence comme ouverte, et non comme le moyen de se fermer en s'enfermant $t^{15}$ " Partant de cette pensée de Carmel Camilleri, nous pouvons dire que Dongala, par l'identité de son personnage revendique un idéal.

\section{L'IDÉAL IDENTITAIRE PRÔNÉ PAR DONGALA}

Lidéal identitaire que semble prôner Dongala est le produit du savoir social. En effet, chacun dans le cours de sa vie, apprend qui il est, par le biais du regard, puis des paroles des autres. Les identités sont d'abord des attributions par autrui. Ainsi le narrateur personnage n'a vu son identité personnelle s'afficher qu'à l'issue de multiples identifications. En procédant de la sorte, Dongala veut signifier que s'identifier est quelque peu synonyme de voyage vers l'autre, mais il ne faut pas rester chez l'autre, "il faut savoir revenir chez soi, si l'on veut forger son identité personnelle ${ }^{16}$." L'idéal ici réside en ce retour aux sources, moyen pour gérer le conflit entre l'unicité et la multiplicité. Tout comme le narrateur, l'idéal identitaire voudrait qu'on soit plusieurs et en même temps soi-même. Et ça, Matapari

15 Carmel Camilleri, "Cultures et stratégies, ou les mille manières de s'adapter " in Revue Sciences Humaines : Lidentité, Lindividu, Le groupe, La société, Auxerre, Éditions Sciences Humaines, 1998, p. 62.

16 Marquer sa différence, entretien avec Pierre TAP, in Revue Sciences Humaines: L'identité, Lindividu, Le groupe, La société, Auxerre, Éditions Sciences Humaines, 1998, p. 65. 
le narrateur personnage a su l'être en demeurant le point d'ancrage traditionnel et scientifique.

À travers lui, Dongala semble montrer qu'en l'Africain d'aujourd'hui, aucune culture ne doit prendre le pas sur les autres. Il suggère dans ce cas un équilibre parfait qui doit faire de chaque Africain un sujet équilibré dans sa société. C'est pourquoi la première phrase du roman de Dongala : " J'AI FAILLI ne pas être né » est sémantiquement chargée. Certes il s’agit de Matapari qui a failli ne pas être né et dont l'identité gémellaire est remise en cause, mais c'est aussi tout Africain colonisé qui a failli ne plus avoir d'identité culturelle. Il s'agit aussi de l'Afrique colonisée tout entière qui a failli ne pas exister culturellement; autrement dit une Afrique dont l'identité a été défigurée, déconstruite voire niée.

Quand le personnage de Dongala, dans un monologue intérieur s'interroge : « les étoiles brillent-elles parce qu'elles sont libres? », nul doute que c'est le vœu de Dongala de voir les Africains recouvrer leur liberté. Ils deviendront ainsi des étoiles pour briller sur le continent africain. En sachant tirer profit des différents actes contradictoires de déconstruction identitaire, ils doivent renaître sous une nouvelle identité. C'est l'image que révèle le paradoxe identitaire lié à l'évolution textuelle du narrateur personnage Matapari.

\section{Conclusion}

La question identitaire telle que présentée dans le roman de Dongala fait comprendre que toute identité est création sociale, processus de déconstruction et de reconstruction. Autrement dit toute identité est toujours en émergence. Elle se construit progressivement au fil du temps. La continuité est dans ce sens le premier élément de l'identité; le deuxième étant la représentation plus ou moins structurée, plus ou moins stable, que l'individu a de lui-même et que les autres se font de lui. Le troisième aspect, c'est l'unicité, c'est-à-dire le sentiment d'être original, de se vouloir différent au point de se percevoir comme unique. À partir de l'exemple de Matapari, il faut donc saisir l'identité personnelle comme l'ensemble des représentations et des sentiments qu'une personne développe à propos d'elle-même. En ce sens, le texte a su présenter Matapari comme une élite de cette conscience identitaire. Et dans cette optique, Dongala le fait percevoir, comme le suggère d'ailleurs le titre de son roman, comme un petit garçon né aussi des étoiles que sont son père (culture scientifique de l'Occident) et son grand-père (culture traditionnelle africaine). 


\section{Bibliographie}

\section{A. 'Texte d'Étude}

Dongala, Boundzeki Emmanuel, Les petits garçons naissent aussi des étoiles, Paris, Les serpents à plumes, 1998.

\section{B. GÉNÉRALitÉS}

Apollinaire, Guillaume, Alcools, Paris, Hatier, 2000.

Camilleri, Carmel, "Cultures et Stratégies, ou les mille manières de S'adapter » in Revue Sciences Humaines : Lidentité, Auxerre, Éditions Sciences Humaines, 1998.

Courtes, Joseph, Analyse Sémiotique du Discours, de l'énoncé à l'énonciation, Paris, Hachette, 1991.

Eliade, Mircéa, Aspects du mythe, Paris, Gallimard, 1963.

Lipiansky, Marc Edmond, "L'identité personnelle » in Revue Sciences Humaines : L'identité, Auxerre, Éditions Sciences Humaines, 1998.

Halen, Pierre, "Constructions identitaires et stratégies d'émergence : notes pour une analyse institutionnelle du système littéraire francophone " in Études françaises, La littérature africaine et ses discours critiques, Montréal, Les Presses de l'Université de Montréal, vol. 37, n², 2001.

Maalouf, Amin, Les identités meurtrières, Paris, Éditions Grasset et Fasquelle, 2006.

Saliba, Jacques, "De l'identité aux identités » in Champ psychosomatique : Les identités, Paris, L’Esprit du Temps, 2001. 